\title{
Verification of Clinical Guidelines Encoded Into Knowledge-Based DSSs
}

\author{
Eugenio Cesario, Massimo Esposito, Giuseppe De Pietro, Domenico Talia \\ Institute for High Performance Computing and Networking (ICAR) \\ National Research Council of Italy (CNR) \\ Italy \\ cesario@icar.cnr.it,massimo.esposito@na.icar.cnr.it,giuseppe.depietro@na.icar.cnr.it,talia@deis.unical.it
}

\begin{abstract}
The empowerment of medical practices, in terms of consistency, effectiveness and efficiency, is being more and more pushed by the encoding of clinical practice guidelines in advanced decision support systems (DSSs). A prerequisite for their wide application is the guarantee of a high level of maintainability and reliability, with respect to different kinds of structural anomalies which can occur in the encoded guidelines and could compromise their practical value. This paper presents a verification approach, based on a formal taxonomy, devised to check the reliability of clinical guidelines encoded as rules. Its final goal is determining two categories of potential structural anomalies, i.e. inconsistency or redundancy, in a very general and understandable fashion. The approach has been developed within the EU IST CHRONIOUS Project, devoted to define a generic platform for remotely monitoring the health status of chronic patients. A sample set of clinical rules pertaining a specific chronic pathology, i.e. Chronic Kidney Disease, has been considered for its initial validation, showing its feasibility and efficacy in a real healthcare case.
\end{abstract}

Keywords-component; Clinical Guidelines, Rule Verification, Rule-based Formalisms, Knowledge-based Decision Support Systems

\section{INTRODUCTION}

In the latest years, clinical practice guidelines have been shown to significantly promote more consistent, effective, and efficient medical practices and improve health outcomes when followed [1]. Several recent studies have suggested that an actual empowerment in the overall quality of care can be realized only if clinical practice guidelines are encoded as advanced decision support systems (in the following, DSSs), i.e. computer-based systems designed to help doctors in making decisions by providing motivated suggestions [2]. Existing DSSs are arranged as knowledge-based systems, i.e. they encode clinical guidelines that incorporate the best scientific evidence of effectiveness with experts' opinions [3].

Actually, representing guidelines into a Knowledge Base (in the following, KB) as a set of rules expressed as condition-action pairs is the most adopted and up-to-date methodology for granting understandability, especially for a non-specialized audience (e.g. clinicians). Each condition-action pair describes possible medical conditions and signs with the appropriate recommendations [4]. Moreover, whether new scientific evidence of effectiveness (combined with opinions of experts) [3] becomes relevant, users (e.g. clinicians) can extend the KB by adding new rules (or editing yet stored ones) according to their daily experience. However, the update of a $\mathrm{KB}$ is a delicate task, since, as shown in some studies [4, 5], clinical rules inserted by several experts with different expertise by clinicians or frequently updated depending on new medical progresses can embody different kinds of structural anomalies, such as inconsistency or redundancy, that compromise their practical value [6]. Hence, structural anomalies in the clinical rules can frequently occur, even for groups looking at the same scientific evidence and using defensible expert judgment procedures, and can undermine confidence in the guideline concept [7].

Such a issue highlights the need for intelligent knowledge management mechanisms for automatically verifying the overall consistency each time the $\mathrm{KB}$ changes. In detail, when guidelines are represented as rules in the $\mathrm{KB}$, they can be formally verified in order to detect structural anomalies that can arise while transferring expertise from a clinician to a DSS and, thus, ensure both the correct behavior and performance of the final DSS.

Taking into account such considerations, this paper presents a verification approach, based on a formal taxonomy, for checking the reliability of clinical guidelines, encoded in the form of rules, with the final aim of determining two categories of potential structural anomalies, i.e. inconsistency or redundancy. Moreover, the strength of the detection (i.e. how much the system is confident about the anomaly identified) and the pattern verified (i.e. the kind of relationship existing among the antecedents of the pair of rules evaluated) are computed. The approach has been developed within the EU IST CHRONIOUS Project [8], which is aimed at defining a generic platform for remotely monitoring the health status of patients suffering from chronic diseases [8,9]. It has been designed in a general way to allow the guideline verification with respect to any disease, independently of the rule representation format used. The remainder of the paper is organized as follows. In Section II an overview of existing verification methods is reported. Section III presents the proposed taxonomy for verifying the reliability of clinical guidelines. A clinical scenario for validating the approach is diffusely described in Section IV. A brief discussion concludes the paper in Section V.

\section{BACKGROUND AND RELATED WORK}

In general, four kinds of structural anomalies, namely redundancy, inconsistency, incompleteness, and circularity 
may generate errors in a set of formal rules [10]. More in detail, a redundancy occurs when two rules have the same set of conditions (even arranged in different order) and the same conclusion. Moreover, two rules are inconsistent if their condition parts are equivalent, but the conclusions are conflicting. Also, circularity refers to a circular dependency between two or more rules. Finally, a set of rules is incomplete when a condition (conclusion) is unmatched by another conclusion (condition). These kinds of anomalies could produce errors, e.g. incorrect or undesired actions, or deteriorate the system performance. However, such anomalies can be automatically detected only from a structural point of view and they require a final check by a domain expert (that is responsible of the final decision regarding which one represents a real error).

In contrast to the intensive efforts made to develop decision support implementations based on clinical guidelines [11], the issue of providing verification mechanisms has been widely neglected thus far. Looking for the domain of knowledge-based systems, where some parallels to the case of clinical knowledge-based DSSs can be found, a substantial number of verification approaches have been proposed for detecting anomalies in rule-bases. In more detail, earlier methods have been devised to locally check the structural and logical integrity of knowledge-based systems, by mainly focusing on detecting errors by pair-wisely verifying rules related to a single conclusion, or belonging to a particular decision making context [4,5]. Differently, in the last two decades, verification techniques have been centered on detecting structural anomalies caused from applying multiple rules in longer inference chains, so being forced to investigate and check the whole rule-base. The majority of such global methods have been based on some graphical notations, i.e. graphs [12,13] or Petri nets [14, 15]. Related research based on Petri nets has been also carried out for checking active rule bases (i.e. rules which define the relationship between events and actions) [16]. Recently, a few methods $[17,18]$ using both static and dynamic model checking have been proposed to globally verify inconsistency and circulation of rule-bases. Furthermore, a DNA-based computing algorithm has been lately applied to the rule-base verification in order to globally detect structural anomalies with efficient computation [19].

Within the CHRONIOUS Project, only inconsistency and redundancy have been considered for being detected, since condition-action guidelines of chronic diseases are essentially represented as 1-level rules without chaining. Condition-action guidelines to be checked can assume a simple structure, (i.e., including comparison operators in their conditions), or a more complex form including statistical or trend patterns (i.e., arithmetical expressions in their conditions). Clinicians involved in the project have expressly required a verification method able to explain why an anomaly is detected in a set of rules, so improving its acceptance and usability in medical settings. As a consequence of such requirements, aforementioned methods proposed in literature have been judged not adequate to this medical context due to their lack of generality, since complex rules including statistical or trend patterns cannot be checked at all, and their poor understandability, since they rely on algorithms and methods that simply provide answers with no further explanation, and clinicians are poorly confident in such black-box systems. Summarizing, all these issues represent the rationale for the approach proposed in this work, which has been expressly thought for a medical context, by realizing a deep and explanatory verification of different forms of condition-action clinical guidelines.

\section{CliniCAL GUIDELINES AND ANOMALY CLASSIFICATION}

This section presents a formal definition of the clinical rules adopted in the CHRONIOUS Project, as well as a description of the taxonomy designed for the anomaly classification between two rules. In particular, Section III.A describes the rule structure, detailing its format. Moreover, it is explained how some clinical guidelines (expressed in medical language) are translated into their corresponding rule encoding. Then, Section III.B introduces the anomaly classification devised for this work and describes the attributes exploited to classify an anomaly instance. Finally, Section III.C presents a formal description of the anomalies as well as handling strategies associated to them.

\section{A. Clinical guidelines and their rule-encoding}

In order to support a deep verification of clinical guidelines for monitoring chronic patients, our study is based on a very expressive rule structure, which includes (in their conditions) comparison operators as well as arithmetical operators. In more detail, each rule is defined as made of one or more antecedent conditions concatenated by logical conjunctive operators, which can be evaluated to be either true or false. Disjunction is not supported since antecedent conditions concatenated by logical disjunctive operators can be remodeled in a semantically equivalent way by using multiple rules. Moreover, each rule has exactly one conclusion.

Rule definition. A rule is a simple conditional declaration that links a logical combination of antecedent conditions to a consequent, according to the following "if-then" structure:

\section{IF antecedents THEN consequent}

whose antecedents and consequent parts are formalized as follows.

Let $A=\left\{a_{1}, \ldots, a_{N}\right\}$ be a set of antecedent attributes and let $C=\left\{c_{l}, \ldots, c_{M}\right\}$ be a set of consequent attributes.

Formally, the antecedent part is a conjunction of $K$ triples in the form $\left\{\operatorname{expr}_{i 1}(A), \operatorname{op}_{i}, \operatorname{expr}_{i 2}(A)\right\}($ for $i=1, \ldots, K)$, i.e.:

antecedents: [ $\left.\quad \operatorname{expr}_{11}(A) \operatorname{op}_{1} \operatorname{expr}_{12}(A)\right]$

$A N D\left[\operatorname{expr}_{21}(A) \operatorname{op}_{2} \operatorname{expr}_{22}(A)\right] \ldots$

$A N D\left[\operatorname{expr}_{K 1}(A) \operatorname{op}_{K} \operatorname{expr}_{K 2}(A)\right]$

where

- $o p_{i}$ is a comparison operator $\{=, \neq,<,>, \leq, \geq\}$,

- $\quad \operatorname{expr}_{i 1}(A)$ and $\operatorname{expr}_{i 2}(A)$ can be :

- a simple antecedent attribute $a_{n} \epsilon A$, with $n$ assuming a value from 1 to $\mathrm{N}$

- a numeric/string value $r$

- a more complex arithmetical expression made of one (or more) attribute( $s) a_{n} \in A$, arithmetical operators $\{+,-, *, /\}$, and numeric/string values. 
The consequent part is an attribute-value pair $\left(c_{m}, v\right)$, connected by an assignment operator, representing the result of the rule, i.e.:

consequent : $\left(c_{m}\right.$ op $\left.v\right)$

where

- $\quad o p$ is an assignment operator

- $c_{m} \in C$ is a simple consequent attribute, with $m$ assuming a value from 1 to $\mathrm{M}$

- $\quad v$ is a numeric or textual value (it is imposed that, when $v$ is defined as textual, the set of admissible values it can assume is only composed of mutually exclusive elements).

According to this structure, rules can be very simple in the cases the antecedents are a conjunction of simple triples in the form $\left(a_{n} o p r\right)$ and only comparison operators are used. For example, Table I describes a simplified version (for the sake of clarity) of the clinical guideline for classifying the Stage 1 of the Chronic Kidney Disease (CKD) [20]. It is first reported in natural language and then encoded according to the proposed rule structure (a symbolic approach has been adopted, where the relevant concepts in the guidelines are modeled as self-explicative attributes, i.e. semantically defined by means of their names).

TABLE I. A SIMPLE EXAMPLE OF CLINICAL GUIDELINE EXPRESSED IN THE RULE STRUCTURE PROPOSED

\begin{tabular}{|c|l|}
\hline $\begin{array}{c}\text { Clinical } \\
\text { guideline: }\end{array}$ & $\begin{array}{l}\text { Individuals with estimated Glomerular Filtration Rate } \geq 90 \\
m L / m i n / 1.73 \mathrm{~m}^{2} \text { and other evidence of chronic kidney } \\
\text { damage (e.g. Persistent Proteinuria) are classified as being } \\
\text { in Stage } 1 .\end{array}$ \\
\hline Rule & $\begin{array}{l}\text { IF (evidenceOfChronicDamage }=\text { "PersistentProteinuria") } \\
\text { AND (estimatedGlomerularFiltrationRate } \geq 90.00) \\
\text { encoding: }\end{array}$ \\
THEN PatientStage $=1$ "
\end{tabular}

Antecedent attributes: evidenceOfChronicDamage, estimatedGlomerularFiltrationRate; consequent attributes: PatientStage.

Moreover, rules can assume also a more complex structure. For example, such a case occurs when the antecedents are a conjunction of expressions in the form $\left\{\operatorname{expr}_{i 1}(A), \operatorname{op}_{i}, \operatorname{expr}_{i 2}(A)\right\}($ for $i=1, \ldots, K)$ and, in addition to comparison operators, arithmetical operators are used in the expressions $\operatorname{expr}_{i 1}(A)$ and $\operatorname{expr}_{i 2}(A)$. Table II illustrates a simplified version of a clinical guideline, defined by clinicians involved in the project, for identifying the presence of fluid overload in patients affected by CKD.

TABLE II. A MORE COMPLEX EXAMPLE OF CLINICAL GUIDELINE EXPRESSED IN THE RULE STRUCTURE PROPOSED

\begin{tabular}{|c|l|}
\hline $\begin{array}{c}\text { Clinical } \\
\text { guideline: }\end{array}$ & $\begin{array}{l}\text { Individuals with systolic blood pressure }>140 \mathrm{mmHg} \text { and a } \\
\text { gain of body weight in three consecutive days }>4 \% \text { are in } \\
\text { an abnormal condition due to Fluid Overload. }\end{array}$ \\
\hline $\begin{array}{c}\text { Rule } \\
\text { encoding: }\end{array}$ & $\begin{array}{l}\text { IF (systolicBloodPressure }>140 \text { ) } \\
\text { AND (bodyWeight }>\text { bodyWeight3DaysBefore * 1.04) } \\
\text { THEN PatientCondition= "Fluid Overload" }\end{array}$ \\
\hline
\end{tabular}

Antecedent attributes: systolicBloodPressure, bodyWeight, bodyWeight3DaysBefore; consequent attribute: PatientCondition.

\section{B. The proposed classification of anomalies}

As introduced into Section II, the verification of clinical guidelines in the context of the CHRONIOUS Project is finalized at detecting the category of two types of anomalies, namely inconsistency and redundancy. Moreover, such a classification is made more complete by other information related to the detected anomaly: the strength and the pattern. The strength is a simple twovalued measure, namely definite or possible, which represents the confidence that a potential anomaly is truly an anomaly. The pattern is a five-valued measure, i.e. equality, subsumption, generalization, non-empty intersection and empty intersection, describing the relationship among the antecedents of the couple of rules evaluated. Summarizing, the triple <pattern, strength, category> fully describes the anomaly that has been detected. While the category is determined by evaluating the consequent parts of the rules examined, the strength and the pattern are detected on the basis of the antecedent parts of the rules evaluated. A description of the proposed taxonomy of anomalies, detailing the meanings of pattern, strength and category, is outlined in the following.

\section{Pattern.}

The pattern of an anomaly specifies the relationship among the antecedents of the couple of rules evaluated. It can assume five different values, whose meanings are detailed as follows:

- Equality: A rule $\mathrm{X}$ is said to be equal to the rule $\mathrm{Y}$ if the antecedent conditions of $\mathrm{X}$ are the same contained in $\mathrm{Y}$.

- Subsumption: A rule $\mathrm{X}$ is said to subsume a rule $\mathrm{Y}$ if the antecedent conditions of $\mathrm{X}$ are a more restrictive superset of the ones contained in $\mathrm{Y}$, (i.e. the subsuming rule $(\mathrm{X})$ contains the same antecedent conditions of the other rule (Y), plus other more specific conditions).

- Generalization: A rule $\mathrm{X}$ is said to generalize a rule $\mathrm{Y}$ if the antecedent conditions of $\mathrm{X}$ are a less restrictive subset of the ones contained in $\mathrm{Y}$.

- Non-empty Intersection: A rule $\mathrm{X}$ is said to not emptily intersect the rule $\mathrm{Y}$ if a subset of the antecedent conditions of $\mathrm{X}$ is also a subset of the ones contained in $\mathrm{Y}$, (i.e. the first rule partially contains the same antecedent conditions of the second one, plus other specific conditions).

- Empty Intersection: A rule $\mathrm{X}$ is said to emptily intersect the rule $\mathrm{Y}$ if the antecedent conditions of $\mathrm{X}$ and $\mathrm{Y}$ are completely different between them.

Depending on these different patterns, the existence of an anomaly can be verified with respect to a pair of rules by calculating a measure of compatibility for each couple of homologous antecedent conditions. A single couple of antecedent conditions is defined as compatible when the respective attributes assume $i$ ) identical values in the case of textual or boolean data types (i.e. an exact match is satisfied) or ii) comparable values in the case of numerical data types (i.e. there is an exact match with couple of single numerical values or the inclusion between intervals of numerical values). Differently, a single couple of antecedent conditions is defined as partial-compatible when it contains different arithmetical expressions and could assume comparable values only for specific values 
assumed by the antecedent attributes involved.

\section{Strength.}

The strength represents the confidence that a potential anomaly is really an anomaly. It can assume two-values, i.e. definite or possible, as described in the following:

- Definite: a potential anomaly is labeled as definite, i.e. it can be detected undoubtedly, in the cases when guidelines expressed in the form of rules contain the same antecedent conditions (i.e. the pattern of equality is verified) or a more/less restrictive superset/subset (i.e. the pattern of subsumption /generalization is verified), the common set of antecedent conditions is compatible and the consequent attributes in their conclusions are the same.

- Possible: a potential anomaly is classified as possible, i.e. it cannot be detected with a full certainty, in three different situations:

- when rules contain the same antecedent conditions or a more/less restrictive superset/subset, the common set of antecedent conditions is partial-compatible and the consequent attributes in their conclusions are the same. In this case, anomalies might occur for particular values assumed by the antecedent attributes involved into the conditions of the couple of rules evaluated;

- when the antecedent conditions are partially the same, the common set of antecedent conditions is compatible or partial-compatible, and the consequent attributes in their conclusions are the same. In this case, anomalies might occur, for instance, for particular values assumed by the remaining antecedent attributes involved into the conditions that are not shared;

- when guidelines are not comparable at all with respect to their antecedent parts, whereas the consequent attributes in their conclusions are the same. In this case, anomalies might occur, for instance, for particular values assumed by the different antecedent attributes involved into the conditions of each rule evaluated.

\section{Category.}

The category completes the classification of the rule anomaly under investigation. In particular, it can assume two values, i.e. inconsistency and redundancy, according to the value assumed by the conclusions of the couple of rules evaluated. In particular:

- Inconsistency: an anomaly is categorized as inconsistency if the consequent attributes of the rules assume different values;

- Redundancy: an anomaly is categorized as redundancy if the consequent attributes of the rules assume identical values.

\section{Formal description of the anomalies and handling strategies.}

In the following all these situations of potential anomalies are discussed in more detail by means of formal definitions and handling strategies associated to them. Indeed, according to the classification described in the previous section, each potential anomaly can be annotated with a handling strategy consisting in a list of admissible actions, which can be executed on the couple of rules containing the anomaly.

The following notation will be used in the rest of the section to formally define potential anomalies involving a couple of rules $X$ and $Y$, where the rule $X$ is new and has to be inserted into the $\mathrm{KB}$ and the rule $Y$ is already stored into the KB:

- Cond $_{x}=\left\{\operatorname{cond}_{x}(n)\right\} n=1, \ldots, N_{x}$ is the set composed by the $N_{x}$ antecedent conditions $\operatorname{cond}_{x}(n)$ used in $X$;

- Cond $_{y}=\left\{\operatorname{cond}_{y}(n)\right\} n=1, \ldots, N_{y}$ is the set composed by the $N_{y}$ antecedent conditions $\operatorname{cond}_{y}(n)$ used in $Y$;

- Conc $_{x}=\left(c_{x}, v_{x}\right)$ is the conclusion (i.e. the consequent attribute $c_{x}$ and its value $v_{x}$ ) used in $X$;

- Conc $_{y}=\left(c_{y}, v_{y}\right)$ is the conclusion (i.e. the consequent attribute $c_{y}$ and its value $v_{y}$ ) used in $Y$;

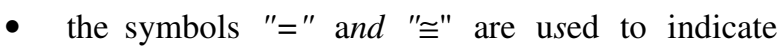
respectively the compatibility and partialcompatibility between two antecedent conditions $\operatorname{cond}_{x}(n)$ and $\operatorname{cond}_{y}(n)$.

\section{$\underline{\text { Definitely inconsistent } \mid \text { redundant anomalies }}$}

A potential anomaly is definitely inconsistent (or definitely redundant) when

- $\operatorname{Conc}_{x} \neq$ Conc $_{y} \quad\left(\right.$ Conc $_{x}=$ Conc $\left._{y}\right) \leftrightarrow c_{x}=c_{y}$ and $v_{x} \neq$ $v_{y}\left(v_{x}=v_{y}\right)$

and one of the following patterns is verified:

- $\quad$ equality: $\operatorname{Cond}_{x}=\operatorname{Cond}_{y} \leftrightarrow \operatorname{cond}_{x}(n)=\operatorname{cond}_{y}(n)$, $n=1, \ldots, N \quad\left(\right.$ where $\left.N=N_{x}=N_{y}\right)$

- subsumption: Cond $_{x} \supset$ Cond $_{y} \leftrightarrow \operatorname{cond}_{x}(n)=$ $\operatorname{cond}_{y}(n), n=1, \ldots, N_{y} \quad\left(\right.$ where $\left.N_{x}>N_{y}\right)$

- generalization: $\operatorname{Cond}_{x} \subset \operatorname{Cond}_{y} \leftrightarrow \operatorname{cond}_{x}(n)=$ $\operatorname{cond}_{y}(n), n=1, \ldots, N_{x} \quad\left(\right.$ where $\left.N_{x}<N_{y}\right)$

Anomaly handling. In the case of detection of one or more definitely inconsistent anomalies after the insertion of the new rule $X$, this latter is not allowed to be saved in the KB. Contextually, the user intervention is required to eliminate the undesired effects caused by it. Differently, in the case of detection of one or more definitely redundant anomalies after the insertion of the rule $X$, this latter can be saved in the KB. The user intervention, even if not strictly required, is suggested to improve the DSS's performance.

\section{$\underline{\text { Possible inconsistent } \mid \text { redundant anomalies }}$}

A potential anomaly is possible inconsistent (or possible redundant) when: 
- $\quad$ Conc $_{x} \neq$ Conc $_{y} \quad\left(\right.$ Conc $_{x}=$ Conc $\left._{y}\right) \leftrightarrow c_{x}=c_{y}$ and $v_{x} \neq$ $v_{y}\left(v_{x}=v_{y}\right)$

and one of the following patterns is verified:

- $\quad$ equality: $\operatorname{Cond}_{x} \cong \operatorname{Cond}_{y} \leftrightarrow \operatorname{cond}_{x}(n) \cong \operatorname{cond}_{y}(n)$, $n=1, \ldots, N \quad\left(\right.$ where $\left.N=N_{x}=N_{y}\right)$

- subsumption: $\operatorname{Cond}_{x} \supset \operatorname{Cond}_{y} \leftrightarrow \operatorname{cond}_{x}(n) \cong$ $\operatorname{cond}_{y}(n), n=1, \ldots, N_{y} \quad\left(\right.$ where $\left.N_{x}>N_{y}\right)$

- $\quad$ generalization: $\operatorname{Cond}_{x} \subset \operatorname{Cond}_{y} \leftrightarrow \operatorname{cond}_{x}(n) \cong$ $\operatorname{cond}_{y}(n), n=1, \ldots, N_{x} \quad\left(\right.$ where $\left.N_{x}<N_{y}\right)$

- $\quad$ non-empty intersection: Cond $_{x} \cap \operatorname{Cond}_{y} \neq \varnothing \leftrightarrow$ $\operatorname{cond}_{x}(n) \cong \operatorname{cond}_{y}(n), n=1, \ldots, N \quad\left(\right.$ where $N<N_{x}$ and $N<N_{y}$ )

- empty intersection: $\operatorname{Cond}_{x} \cap \operatorname{Cond}_{y}=\varnothing$

Anomaly handling. In the case of detection of one or more possible inconsistent or redundant anomalies after the insertion of the new rule $X$, this latter is allowed to be saved in the KB. The user intervention, even if not strictly required, is suggested to improve the DSS's performance.

\section{AN APPLICATIVE SCENARIO: CKD GUIDELINES}

This section describes a real case the proposed taxonomy has been applied on, aimed at detecting and classifying anomalies occurring on a sample set of clinical guidelines selected from the literature or specified by the clinicians involved into the CHRONIOUS Project. In particular, the analysis has been focused on a specific clinical pathology, i.e. the CKD. The rule verification has been implemented as Web service (according to the Service Oriented Architecture), i.e. ConsistencyCheckerWS. This module has been applied for validating the approach by a practical case, as a proof of concept. In particular, we have selected two clinical recommendations (encoded as rules in Table III) and checked possible anomalies within a given rule set stored in the KB.

TABLE III. THE TWO RULES TO BE VERIFIED

\begin{tabular}{|c|l|}
\hline ID & \multicolumn{1}{|c|}{ Rule Formalization } \\
\hline A & $\begin{array}{l}\text { IF (evidenceOfChronicDamage }=\text { "PersistentProteinuria") AND } \\
\text { (estimatedGlomerularFiltrationRate } \geq 90.00) \\
\text { THEN PatientStage }=\text { "1" }\end{array}$ \\
\hline B & $\begin{array}{l}\text { IF (systolicBloodPressure > 140) AND (bodyWeight }> \\
\text { bodyWeight3DaysBefore * 1.04) THEN PatientCondition= "Fluid } \\
\text { Overload" }\end{array}$ \\
\hline
\end{tabular}

Antecedent attributes: evidenceOfChronicDamage, estimatedGlomerularFiltrationRate, estimatedGlomerularFiltrationRate3DaysBefore, systolicBloodPressure, diastolicBloodPressure bodyWeight, bodyWeight3DaysBefore; consequent attributes: PatientStage, PatientCondition (where "Fluid Overload" and "Arterial Hypertension" are its mutually exclusive values).

The rule set composing the whole $\mathrm{KB}$ is reported in Table IV. It includes 16 rules, some having the antecedents part in a very simple structure and others in a more complex form. For example, some rules have conditions with only comparison operators (i.e., Rules 1-6), while others (i.e., Rules 7-9) with also arithmetical expressions. For the sake of clarity, such rules have been purposelyinserted with the aim of generating different kinds of anomalies with respect to the Rule A and Rule B and, consequently, evaluating the effectiveness and feasibility of the approach. For such a reason, it is not relevant if some anomalies are already present in the KB.

TABLE IV. THE RULE SET COMPOSING THE KB

\begin{tabular}{|c|c|}
\hline ID & Rule Formalization \\
\hline 1 & $\begin{array}{l}\text { IF (evidenceOfChronicDamage }=\text { "PersistentProteinuria") AND } \\
\text { (estimatedGlomerularFiltrationRate } \geq 90.00 \text { ) THEN PatientStage }=" 2 "\end{array}$ \\
\hline 2 & $\begin{array}{l}\text { IF }(\text { evidenceOfChronicDamage }=\text { "PersistentProteinuria") THEN } \\
\text { PatientStage }=\text { "2" }\end{array}$ \\
\hline 3 & $\begin{array}{l}\text { IF (evidenceOfChronicDamage }=" \text { PersistentProteinuria") AND } \\
\text { (estimatedGlomerularFiltrationRate3DaysBefore } \geq 90.00 \text { ) AND } \\
\text { (estimatedGlomerularFiltrationRate } \geq 90.00 \text { ) THEN } \text { PatientStage }=\text { " } 2 "\end{array}$ \\
\hline 4 & $\begin{array}{l}\text { IF (evidenceOfChronicDamage }=" \text { PersistentProteinuria") AND } \\
\text { (estimatedGlomerularFiltrationRate } \geq 90.00 \text { ) } \mathbf{T H E N} \text { PatientStage }=" 1 "\end{array}$ \\
\hline 5 & $\begin{array}{l}\text { IF (evidenceOfChronicDamage }=\text { "PersistentProteinuria") } \\
\text { THEN PatientStage }=" 1 "\end{array}$ \\
\hline 6 & $\begin{array}{l}\text { IF (evidenceOfChronicDamage }=\text { "PersistentProteinuria") AND } \\
\text { (estimatedGlomerularFiltrationRate3DaysBefore } \geq 90.00 \text { ) AND } \\
\text { (estimatedGlomerularFiltrationRate } \geq 90.00 \text { ) THEN PatientStage }=" 1 "\end{array}$ \\
\hline 7 & $\begin{array}{l}\text { IF (systolicBloodPressure }>140 \text { ) AND } \text { (bodyWeight }> \\
\text { bodyWeight } 3 \text { DaysBefore } * 1.14 \text { ) THEN PatientCondition= "Arterial } \\
\text { Hypertension" }\end{array}$ \\
\hline 8 & $\begin{array}{l}\text { IF (bodyWeight }>\text { bodyWeight } 3 \text { DaysBefore }+10 \text { ) } \\
\text { THEN PatientCondition= "Arterial Hypertension" }\end{array}$ \\
\hline 9 & $\begin{array}{l}\text { IF (systolicBloodPressure > 140) AND (diastolicBloodPressure >90) } \\
\text { AND (bodyWeight }>\text { bodyWeight3DaysBefore }+10) \\
\text { THEN PatientCondition= "Arterial Hypertension" }\end{array}$ \\
\hline 10 & $\begin{array}{l}\text { IF (systolicBloodPressure > 140) AND (diastolicBloodPressure >90) } \\
\text { THEN PatientCondition= "Arterial Hypertension" }\end{array}$ \\
\hline 11 & $\begin{array}{l}\text { IF (diastolicBloodPressure > 90) THEN PatientCondition= "Arterial } \\
\text { Hypertension" }\end{array}$ \\
\hline 12 & $\begin{array}{l}\text { IF (systolicBloodPressure > 140) AND (bodyWeight }> \\
\text { bodyWeight3DaysBefore * 1.14) THEN PatientCondition= "Fluid } \\
\text { Overload" }\end{array}$ \\
\hline 13 & $\begin{array}{l}\text { IF (bodyWeight }>\text { bodyWeight3DaysBefore }+10 \text { ) } \\
\text { THEN PatientCondition= "Fluid Overload" }\end{array}$ \\
\hline 14 & $\begin{array}{l}\text { IF (systolicBloodPressure > 140) AND (diastolicBloodPressure >90) } \\
\text { AND (bodyWeight }>\text { bodyWeight3DaysBefore }+10 \text { ) THEN } \\
\text { PatientCondition= "Fluid Overload" }\end{array}$ \\
\hline 15 & $\begin{array}{l}\text { IF (systolicBloodPressure > 140) AND (diastolicBloodPressure > 90) } \\
\text { THEN PatientCondition= "Fluid Overload" }\end{array}$ \\
\hline 16 & $\begin{array}{l}\text { IF (diastolicBloodPressure > 90) THEN PatientCondition= "Fluid } \\
\text { Overload" }\end{array}$ \\
\hline
\end{tabular}

Antecedent attributes: evidenceOfChronicDamage, estimatedGlomerularFiltrationRate, estimatedGlomerularFiltrationRate3DaysBefore, systolicBloodPressure, diastolicBloodPressure bodyWeight, bodyWeight3DaysBefore; consequent attributes: PatientStage, PatientCondition (where "Fluid Overload" and "Arterial Hypertension" are its mutually exclusive values).

The set of rules shown in Table IV has been verified with respect to the new ones, i.e. the rule $\mathrm{A}$ and $\mathrm{B}$, so detecting and classifying the anomalies, as summarized in Table V. In particular, 16 different anomalies have been pointed out, whose descriptions in terms of category, pattern and strength are detailed in the table.

Summarizing, as it can be seen, the proposed approach has shown to be an innovative and valuable way to verify the consistency of clinical guidelines, if encoded as rules, in real clinical case studies. 


\section{ACKNOWLEDGMENT}

\begin{tabular}{|c|c|c|c|c|}
\hline Anomaly & Category & Pattern & Strength & Rules \\
\hline $\mathbf{1}$ & Inconsistency & Equality & Definite & $A, 1$ \\
\hline $\mathbf{2}$ & Inconsistency & Subsumption & Definite & $A, 2$ \\
\hline $\mathbf{3}$ & Inconsistency & Generalization & Definite & $A, 3$ \\
\hline $\mathbf{4}$ & Redundancy & Equality & Definite & $A, 4$ \\
\hline $\mathbf{5}$ & Redundancy & Subsumption & Definite & $A, 5$ \\
\hline $\mathbf{6}$ & Redundancy & Generalization & Definite & $A, 6$ \\
\hline $\mathbf{7}$ & Inconsistency & Equality & Potential & $B, 7$ \\
\hline $\mathbf{8}$ & Inconsistency & Subsumption & Potential & $B, 8$ \\
\hline $\mathbf{9}$ & Inconsistency & Generalization & Potential & $B, 9$ \\
\hline $\mathbf{1 0}$ & Inconsistency & $\begin{array}{c}\text { Non-empty } \\
\text { Intersection }\end{array}$ & Potential & $B, 10$ \\
\hline $\mathbf{1 1}$ & Inconsistency & Empty Intersection & Potential & $B, 11$ \\
\hline $\mathbf{1 2}$ & Redundancy & Equality & Potential & $B, 12$ \\
\hline $\mathbf{1 3}$ & Redundancy & Subsumption & Potential & $B, 13$ \\
\hline $\mathbf{1 4}$ & Redundancy & Generalization & Potential & $B, 14$ \\
\hline $\mathbf{1 5}$ & Redundancy & $\begin{array}{c}\text { Non-empty } \\
\text { Intersection }\end{array}$ & Potential & $B, 15$ \\
\hline $\mathbf{1 6}$ & Redundancy & Empty Intersection & Potential & $B, 16$ \\
\hline
\end{tabular}

The research reported in this paper was supported by the European Union as part of the CHRONIOUS Project under contract number FP7-ICT-2007-1-216461.

\section{REFERENCES}

[1] I. Scott, What are the most effective strategies for improving quality and safety of health care? Internal Medicine Journal. 39, 6 (2009), pp. 389-400.

[2] E. Shortliffe, J. Cimino,Biomedical informatics: computer applications in health care and biomedicine. Springer-Verlag New York, 2006.

[3] L. Leape, Practice guidelines and standards: an overview. QRB. Quality review bulletin, 16, 2(1990), pp. 42-49.

[4] R. Shiffman, Representation of clinical practice guidelines in conventional and augmented decision tables. J. Amer. Med. Inf. Assoc., 4, 1997, pp. 382-393.

[5] R. Shiffman, R. Greenes. Improving clinical guidelines with logic and decision-table techniques. Medical Decision Making, 14, 3(1994), pp. 245-254.

[6] G. Duftschmid, S. Miksch. Knowledge-based verification of clinical guidelines by detection of anomalies. Artificial intelligence in medicine, 22, 1(2001), pp.23-41.

[7] M.D. Cabana, C.S. Rand, N.R. Powe, A.W. Wu, M.H. Wilson, P.A.C. Abboud, H.R. Rubin, "Why don't physicians follow clinical practice guidelines?", Journal of American Medical Association, 282, 15 (1999), pp. 1458--1465.

[8] CHRONIOUS project's web site: http://www.chronious.eu/

[9] A. Papadopoulos, D. I. Fotiadis, M. Lawo, F. Ciancitto, C. Podolak, R. L. Dellaca, G. Munaro, R. Rosso, Chronious: A Wearable System for the Management of Chronic Disease Patients, in Proc. 9th International Conference on Information Technology and Applications in Biomedicine, Nov. 5-7, 2009.

anomalies purposely-inserted, so proving its feasibility and efficacy. Actually, the Consistency Checker module has been released to the clinicians, within the CHRONIOUS platform, and the validation phase has started for more extensively testing the approach in action.

\section{CONCLUSIONS AND FUTURE WORKS}

This paper presented a verification approach based on a formal taxonomy for checking the reliability of clinical guidelines, encoded in the form of rules, with the final aim of determining two categories of potential structural anomalies, i.e. inconsistency or redundancy. Differently from existing methods or the verification of knowledgebased systems, this approach was specifically thought for a deep verification of medical knowledge. In fact, it was designed to check rules characterized by a very simple structure (i.e., including comparison operators in their conditions), as well as by more complex forms including statistical or trend patterns (arithmetical expressions in their conditions). The approach was devised sufficiently general in order to be applied independently of the guideline representation format used. It was developed within the EU IST CHRONIOUS Project and initially validated on a sample set of clinical rules pertaining CKD, so proving its feasibility and efficacy.

As future work, the approach will be deployed to the clinical sites and its functioning precisely tested by clinicians in the context of CHRONIOUS Project, which is, currently, starting its validation phase. A specific focus in this validation will be given to the two pathologies chosen for project demonstration, namely Chronic Obstructive Pulmonary Disease and Chronic Kidney Disease.

[10] D.L. Nazareth, Issues in the Verification of Knowledge in RuleBased Systems. International Journal of Man-Machine Studies, 30, 1989, pp. 255-271.

[11] J. Brokel, M. Shaw, C. Nicholson. Expert clinical rules automate steps in delivering evidence-based care in the electronic health record. Computers Informatics Nursing, 24, 4(2006), pp.196-205.

[12] G.S. Gursaran, S. Kanungo, and A.K. Sinha, "Rule-Base Content Verification Using a Digraph-Based Modeling Approach," Artificial Intelligence Eng., 13, 1999, pp. 321-336.

[13] C. Wu, S. Lee, "A Token-Flow Paradigm for Verification of RuleBased Expert Systems", IEEE Trans. Syst. Man Cybern. Part B Cybern., 30, 4(2000), pp. 616-624.

[14] V.R.L. Shen, T.T.-Y. Juang, "Verification of Knowledge-Based Systems Using Predicate/Transition Nets", IEEE Trans. Syst. Man Cybern. Part A Syst. Humans, 38,.1(2008) pp.78-87.

[15] X. He, W.C. Chu, H. Yang, "A New Approach to Verify RuleBased Systems Using Petri Nets", Inf. Software Technol., 45, 2003, pp. 663-669.

[16] X. Li, J. Medina, S. Chapa, "Applying Petri nets in active database systems", IEEE Trans. Syst. Man Cybern. Part C Appl. Rev., 37, 4(2007), p. 482-493.

[17] D. Xu, K. Xia, D. Zhang, H. Zhang,"Model Checking the Inconsistency and Circularity in Rule-Based Expert Systems", Int. J. Comput. Inf. Sci., 2, 1(2009), pp. 12-17.

[18] H. Huang, S. Huang, T. Zhang, "A Formal Method for Verifying Production Knowledge Base", in Proc. of the 2009 Fourth International Conference on Internet Computing for Science and Engineering, 2009, pp. 19-23.

[19] Y. Chung-Wei, C. Chih-Ping, "Molecular Verification of RuleBased Systems Based on DNA Computation", IEEE Trans. Knowl. Data Eng., 20, 7(2008), pp. 965-975.

[20] National Kidney Foundation. KDOQI clinical practice guidelines for chronic kidney disease: Evaluation, classification, and stratification. American Journal of Kidney Diseases, 39, S1S266(2002) 